\title{
An Energy-Aware Algorithm for TDMA MAC Protocols in Real-Time Wireless Networks
}

\author{
Gianluca Franchino, Giorgio Buttazzo and Mauro Marinoni \\ firstname.lastname@sssup.it \\ Scuola Superiore S. Anna Pisa, Italy
}

\begin{abstract}
In distributed embedded systems operated by battery, energy management is a critical issue that has to be addressed at different architecture levels. For systems that tightly interact with the environment, an additional goal is to enforce a set of real-time constraints to guarantee a desired performance. A lot of research has focused on power management at the communication level, especially for MAC protocols. However, not many authors considered both real-time and energy requirements in wireless communication systems. In this paper we present ElMAC, an elastic energy-aware algorithm at the MAC level for wireless distributed systems with real-time constraints. Under this framework, each node can adapt its bandwidth requirements to balance performance versus energy consumption, taking both lifetime and message deadlines into account. We describe the algorithm for a generic TDMA MAC protocol.
\end{abstract}

\section{INTRODUCTION}

Embedded systems operated by battery are exponentially increasing, including mobile phones, multimedia players, portable devices, sensor networks, teams of mobile robots, and distributed communication systems. Some of these systems are required to interact with the environment, reading sensors, reacting to events, and performing proper control actions. Consider, for example, a distributed monitoring system consisting of tens or hundreds of mobile nodes delivered in a specific area to detect intrusions, or abnormal situations. To achieve this goal, each node has to be equipped with sensors (to detect the events of interest), motors (to move in the environment), processing units (to analyze data and compute control actions), and communication devices (to coordinate with the other nodes and transmit the information to the base station). From one hand, the interaction with the environment creates implicit timing constraints on the application that have to be enforced on the node activities in order to achieve a desired performance. From the other hand, since nodes are powered by batteries, the available energy must be carefully managed to prolong the system lifetime long enough to complete the mission.

Unfortunately, real-time and energy requirements have contrasting objectives and they cannot be considered separately during system design. For instance, meeting real-time constraints on sensory and control activities would require the onboard processor to use a suitable scheduling algorithm and to run fast enough in order to complete all the tasks within their deadlines. However, running with a high speed causes a higher power consumption, which reduces the node lifetime. Similarly, at the communication level, reducing the end-to-end delay in message delivery would require the nodes to have a large transmission range to guarantee a single hop connection, even though a large transmission range consumes more power, reducing the system autonomy.

To take both requirements into account, the system should reduce power consumption as much as possible to extend its lifetime, but should use enough power to satisfy timing constraints. Hence, the objective of the system is to minimize energy consumption, while guaranteeing timing constraints.

To minimize energy consumption, power should be saved at different architecture levels. At the application level, specific devices can be turned off, or configured at a proper operating low-power mode (if any), when they are not used for a sufficiently long interval of time. At the operating system level, suitable scheduling and resource management algorithms can be adopted to execute tasks at the minimum speed that guarantees the required performance constraints. At the network level, the transmission power of each node can be set at the minimum level that guarantees a given degree of connectivity. Moreover, as transceivers have different working modes (e.g., transmitting, receiving, and sleep mode), a node can set its transceiver on sleep mode to save energy whenever it does not need to communicate.

In the market there exist several transceivers single-chip suitable to build small radio devices. These chips have some smart features that can be exploited to design energy-aware transmission protocols [1], [2]. For instance several devices provide:

- RSSI (Receiving Signal Strength Indicator), which is a value proportional to the strength of the received RF signal. It can give a greedy esteem of the distance from the source, if the transmission power is known.

- Different levels of transmission power. They can be exploited, in conjunction with the RSSI, to save energy, adapting the transmission power to the distance between source and sink nodes.

- Different operating modes, each characterized by a different level of power consumption. The most common are: sleep, receiving, and transmitting.

In this paper, we describe a methodology to save energy at the communication level, precisely at the Medium Access Control (MAC) level, in wireless networks with real-time requirements. As proposed in [3], we take into account the following main sources of energy waste:

- Collision. If a packet is corrupted it has to be resent, therefore both the sender and the receiver have to consume additional energy to exchange the packet.

- Overhearing. This is the energy wasted by a node when 
listening to packets directed to other nodes.

- Control packet overhead. This is the energy used to send and receive control packets, or adding control characters to the packets.

- Idle listening. This is the energy wasted by a node while listening to receive possible traffic that is not sent.

In some application, idle listening can be the main cause of energy waste, since nodes can stay for a long time without sending messages, as the case of a sensor network where no events are generated in the environment.

Beside the energy waste sources described above, in this work we also consider the energy consumed by a transceiver to switch between operating modes. Such an overhead becomes relevant when the time intervals in which a node goes to idle are too short. In fact, in this case, the energy needed to switch from an active mode (transmitting or receiving) to the sleep mode and viceversa, could be greater than that needed to stay always active.

\section{A. Contributions and summary}

In this work, we propose an energy model and a related algorithm to reduce the energy consumption in a wireless network, with the objective of reaching a given system lifetime while guaranteeing periodic messages deadlines. In particular, we present El-MAC, an elastic energy-aware algorithm at the MAC level, where each node can adapt its bandwidth requirements to balance performance versus energy consumption. The proposed methodology is designed for Time Division Multiplexing Access (TDMA) protocols, and for a network traffic generated by real-time streams.

The remainder of the paper is organized as follows. Section II describes the related work. Section III introduces the problem formulation, and describes both the power consumption and the message stream models. Section IV illustrates the El-MAC algorithm and presents an applicative example. Section V analyzes the properties of the algorithm. Section VI presents some simulation results. Finally, Section VII concludes the paper and presents some future developments.

\section{RELATED WORK}

The issues of real-time communication and energy saving over wireless networks have received much attention during the last years. However, not many authors addressed both problems simultaneously. Adamou et al. [4] proposed a MAC protocol (PARMAC) that provides a guaranteed bandwidth for real-time traffic, while reducing the energy consumption. Time is divided in frames of fixed length. Each frame is divided into a Reservation Period (RP) and a Contention Free Period (CFP). During the RP, nodes contend to reserve transmission windows in the CFP; in the CFP, nodes send data packets without contention during their reserved slots, and sleep when they do not have to transmit or receive traffic. The authors highlight that PARMAC minimizes the idle time and allows a node to sleep during a CFP. Moreover, since the collisions are not frequent a node needs to exchange less packets to complete a transmission, thus it needs less energy to deliver a message. However, in this work the authors do not give a method to compute the maximum energy consumption, nor compare the energy performance with respect to other protocols.

Caccamo et al. [5] proposed a cellular network architecture with a MAC protocol based on the Earliest Deadline First algorithm (EDF) [6]. Implicit prioritization is achieved by exploiting the periodic nature of the traffic in sensor networks. The authors analyze the capacity of the network using an implicit EDF scheme, where each node locally generates the same EDF schedule and accesses the channel without collisions. The implementation of this scheme requires clock synchronization among nodes contending a channel. Moreover, slotted reservation may lead to waste of bandwidth if nodes either fail or, due to the variable packet size, do not use their reserved slots entirely. To address these problems, Crenshaw et al. [7] presented a new version of the protocol, called Robust Implicit EDF (RI-EDF), which does not require clock synchronization, providing bandwidth reclamation and robustness in the presence of certain classes of node failures. Furthermore, the authors presented a power-aware extension, based on the partition of nodes in sources and sinks. Sources are nodes which transmit data. Sinks are node interested in the actual data sent; they can also send data but they are still classified as sinks. This extension needs a couple of additions in the protocol. First, to denote whether a packet is from a sink or a source, a new field is added in the packet headers. Second, by means of a periodic beacon packet, a sink may optionally solicit data from the sources.

Unlike the previous protocols which are mainly focused on the MAC layer, SPEED [8] is a protocol designed for realtime communication in sensor networks, which defines the behavior rules for all layers of the communication stack. In particular, SPEED provides a soft (probabilistic) guarantee on the real-time traffic delivering, through a feedback control scheduling mechanism [9]. Energy savings performance is analyzed comparing the SPEED routing algorithm with other routing solutions available in the literature.

Koubaa et al. [10] analyzed the power efficiency and the timeliness of the IEEE 802.15.4 standard under the GTSs mechanism. They also proposed a method to select the protocol parameters, to trade the power efficiency and the delay bound guarantees provided by this technology.

A further interesting work is the RT-Link protocol, proposed by Rowe et al. [11], which is a time-synchronized link layer protocol that guarantees a predictable life time and a bounded end-to-end delay across multiple hops. Other works concerning real-time wireless communication can be found in [12] and [13].

\section{PROBLEM AND MODELS}

In this work we consider a real-time distributed system composed by $n$ computational nodes sharing a communication channel, whose access is managed through a MAC layer protocol, using a TDMA scheduling algorithm.

\section{A. Network traffic model}

The traffic generated by each node $i$ is modeled by a message stream which can be of two kinds: real-time $\left(S_{i}^{R T}\right)$, or bandwidth-guaranteed $\left(S_{i}^{B G}\right)$. The former type of traffic 
is modeled by a periodic/sporadic real-time message stream described by three parameters $\left(C_{i}, T_{i}, D_{i}\right)$, where:

- $C_{i}$ is the maximum amount of time required to transmit a message in the stream.

- $T_{i}$ is the inter-arrival period between consecutive messages in the stream. If the first message in stream $S_{i}$ of node $i$ is generated at time $t_{i, 1}$, then the $j$-th message in stream $S_{i}$ will be generated at time $t_{i, j}=t_{i, 1}+(j-1) T_{i}$, where $j=1,2, \ldots$.

- $D_{i}$ is the relative deadline associated with messages in stream $S_{i}$, that is, the maximum amount of time that can elapse between a message arrival and the completion of its transmission.

The bandwidth-guaranteed traffic is modeled by a stream described by a single parameter $U_{i}^{B G}$ : the channel utilization, namely the bandwidth required by the stream.

For the sake of simplicity, we assume that each node $i$ is associated with a message stream $\left(S_{i}^{R T}\right.$ or $\left.S_{i}^{B G}\right)$, however, it is not difficult to extend the analysis to the case in which a node is associated with more streams. Hence, we consider the communication system composed by a stream set $\Gamma=$ $\left\{S_{1}, S_{2}, \ldots, S_{n}\right\}$, where each stream can be a real-time or a bandwidth-guaranteed stream.

The total channel utilization, $U$, which measures the total demand placed on the system by the stream message set is

$$
U=\sum_{i=1}^{n} U_{i}
$$

where $U_{i}=U_{i}^{R T}=C_{i} / T_{i}$ in case of a real-time stream, or $U_{i}=U_{i}^{B G}$ in case of a bandwidth guaranteed streams.

It is reasonable for several applications to assume that the stream parameters are constant for each message, but there are situations in which periodic messages could be delivered at different rates in different working conditions. The possibility of varying the stream periods makes the communication system more flexible. For instance, in dynamic systems where communication streams can be created and destroyed at run time, whenever a new stream cannot be accepted in the system because its utilization would make the channel occupation greater than 1 , instead of rejecting the stream, the system could try to reduce the other streams utilizations, decreasing the channel occupation in order to accept the new request. The same reasoning can be applied for bandwidth-guaranteed streams.

Elastic scheduling was first proposed in [14] for realtime tasks. We extend this framework to message streams, considering each stream utilization as a spring with a given elastic coefficient, whose length can be modified by changing the maximum message duration $C_{i}$, the period $T_{i}$ or the guaranteed bandwidth $U_{i}^{B G}$.

Without loss of generality, in the rest of the paper we assume that each message stream, real-time or bandwidth guaranteed, is described by three parameters: a minimum required bandwidth $U_{i}^{\min }$, a maximum required bandwidth $U_{i}^{\max }$, and an elastic coefficient $\epsilon_{i}$, which specifies the flexibility of the stream to change its utilization. Therefore, an elastic stream is denoted as: $S_{i}=\left(U_{i}^{\min }, U_{i}^{\max }, \epsilon_{i}\right)$.

\section{B. Energy model}

From now on, $U_{i}$ will denote the actual utilization of stream $S_{i}$, which can vary in the range $\left[U_{i}^{\min }, U_{i}^{\max }\right]$. To compute the average power $P_{i}$ consumed by node $i$, we first observe that each node:

- transmits for a certain amount of time depending on the associated stream parameters. For instance, considering a periodic stream, node $i$ transmits for $C_{i}$ time units every $T_{i}$. In other words, the time the node is on transmission is proportional to the stream bandwidth $U_{i}^{R T}$, or $U_{i}^{B G}$ with bandwidth-guaranteed traffic.

- is in listening mode when the other nodes are transmitting $^{1}$, and it goes on sleep during idle times.

To take switching overhead into account, we assume that each node takes $t_{i}^{s w}$ units of time to switch between two operating modes and consumes a power $P_{i}^{s w}$ during switching (for simplicity, we assume that both $t_{i}^{s w}$ and $P_{i}^{s w}$ are constant and independent from the modes).

Considering also those protocols where nodes exchange control packets to guarantee the network operation, we assume that a node $i$ can make use of a channel bandwidth $U_{i}^{\text {ctrl }}$ to transmit control messages.

Let $P_{i}^{t x}, P_{i}^{r x}$, and $P_{i}^{s l}$ be the power consumed in transmission mode, receiving mode, and sleep mode, respectively. The average power consumed by node $i$ can be written as a weighted sum of the powers consumed by the node on different operating modes, considering that $U_{i}$ is the fraction of time node $i$ stays on transmission, $U-U_{i}$ is the fraction for receiving, $U_{i}^{s w}$ is the fraction spent to switch between modes, $U_{i}^{c t r l}$ is the fraction of time a node stays on transmission to deliver control information, $\sum_{j=1}^{n} U_{j}^{c t r l}-U_{i}^{\text {ctrl }}=U^{\text {ctrl }}-U_{i}^{\text {ctrl }}$ is the fraction for receiving control information from the other nodes, and $1-U-U_{i}^{s w}-U_{i}^{c t r l}$ is the fraction for staying on sleep. Thus, we have:

$$
\begin{aligned}
P_{i} & =U_{i} P_{i}^{t x}+\left(U-U_{i}\right) P_{i}^{r x}+U_{i}^{s w} P_{i}^{s w} \\
& +U_{i}^{c t r l} P_{i}^{t x}+\left(U^{c t r l}-U_{i}^{c t r l}\right) P_{i}^{r x} \\
& +\left(1-U-U^{c t r l}-U_{i}^{s w}\right) P_{i}^{s l}
\end{aligned}
$$

Observing that $U-U_{i}=\sum_{j=1, j \neq i}^{n} U_{j}$, the last equation can be rewritten as follows:

$$
\begin{aligned}
P_{i} & =U_{i} P_{i}^{t x}+\sum_{j=1, j \neq i}^{n} U_{j} P_{i}^{r x}+U_{i}^{s w} P_{i}^{s w} \\
& +U_{i}^{c t r l}\left(P_{i}^{t x}-P_{i}^{r x}\right)+U^{c t r l} P_{i}^{r x} \\
& +\left(1-\sum_{j=1, j \neq i}^{n} U_{j}-U_{i}-U^{c t r l}-U_{i}^{s w}\right) P_{i}^{s l} \\
& =U_{i} P_{i}^{t x}+\sum_{j=1, j \neq i}^{n} U_{j} P_{i}^{r x}+U_{i}^{s w} P_{i}^{s w}+U^{c t r l} P_{i}^{r x} \\
& +U_{i}^{c t r l}\left(P_{i}^{t x}-P_{i}^{r x}\right)+P_{i}^{s l}-\sum_{j=1, j \neq i}^{n} U_{j} P_{i}^{s l} \\
& -U_{i} P_{i}^{s l}-U^{c t r l} P_{i}^{s l}-U_{i}^{s w} P_{i}^{s l}
\end{aligned}
$$

\footnotetext{
${ }^{1}$ Such a pessimistic assumption is made for the sake of simplicity, and will
} be relaxed later. 
and gathering the terms,

$$
\begin{aligned}
P_{i} & =U_{i}\left(P_{i}^{t x}-P_{i}^{s l}\right)+\left(\sum_{j=1, j \neq i}^{n} U_{j}\right)\left(P_{i}^{r x}-P_{i}^{s l}\right) \\
& +U_{i}^{c t r l}\left(P_{i}^{t x}-P_{i}^{r x}\right)+P_{i}^{s l}+U^{c t r l}\left(P_{i}^{r x}-P_{i}^{s l}\right) \\
& +U_{i}^{s w}\left(P_{i}^{s w}-P_{i}^{s l}\right)
\end{aligned}
$$

Now, we define:

$$
\begin{cases}\Delta_{i} & =U_{i}^{c t r l}\left(P_{i}^{t x}-P_{i}^{r x}\right)+P_{i}^{s l}+U^{c t r l}\left(P_{i}^{r x}-P_{i}^{s l}\right) \\ & +U_{i}^{s w}\left(P_{i}^{s w}-P_{i}^{s l}\right) \\ P_{i}^{t s} & =P_{i}^{t x}-P_{i}^{s l} \\ P_{i}^{r s} & =P_{i}^{r x}-P_{i}^{s l} .\end{cases}
$$

Hence, Equation (2) can be written as

$$
P_{i}=U_{i} P_{i}^{t s}+\sum_{j=1, j \neq i}^{n} U_{j} P_{i}^{r s}+\Delta_{i}
$$

From Equation (3), it is clear that the power consumed by node $i$ depends on the node utilization $U_{i}$, on the sum of the utilizations of the other nodes, on $U_{i}^{s w}, U^{\text {ctrl }}$ and $U_{i}^{\text {ctrl }}$.

Notice that, the higher the power consumed in sleep mode, the lower $P_{i}^{t s}$ and $P_{i}^{r s}$; that is, the lower the contribution given by the node utilizations on the power consumption. The worst case happens when $P_{i}^{t x} \simeq P_{i}^{r x} \simeq P_{i}^{s l}$, then $P_{i}=P_{i}^{s l}+$ $U_{i}^{s w}\left(P_{i}^{s w}-P_{i}^{s l}\right)$, that is the node is uselessly wasting power to switch between operating modes.

If $t$ is the time elapsed from the system start-up, let $P_{i}(t)$ be the power dissipated by node $i$ at a time $t$, for sufficiently large $t$ the energy $E_{i}(t)$ consumed in the worst case by node $i$ at time $t$ is:

$$
E_{i}(t)=\int_{0}^{t} P_{i}(t) d t \simeq P_{i} \cdot t .
$$

The network lifetime is the time span from the system startup to the instant when the network is considered nonworking. However, when a network is considered nonworking depends on the application [15], [16].

In this work we consider the network lifetime as the time of the first node failure due to energy exhaustion. More precisely, if $E_{i}^{0}$ is the energy available at node $i$ at time $t=0$ and $L_{i}$ is its lifetime, the network lifetime $L_{n e t}$ is defined as:

$$
L_{n e t}=\min _{i}\left(L_{i}\right)=\min _{i}\left(\frac{E_{i}^{0}}{P_{i}}\right) .
$$

Analyzing the energy model expressed by Equation (3), it should be clear that the model is very pessimistic, because it assumes that a node remains in a receiving state also when a message is sent to another destination. In this case the energy is wasted due to overhearing (see Section I). To reduce such an energy waste, a node that starts receiving a message directed to a different destination, could extract the message length from the header, and could switch to sleep for the message duration. It turns out that it is difficult to take such energy into account saving in the energy model, as in several applications a node cannot know in advance when a stream does not send traffic to it. However, if such an info is available, the node can better esteem its energy consumption reducing the energy wasted for overhearing. For instance, if $\widehat{\mathbf{S}}$ is the set of streams that do not send messages to node $i$, such a node can improve its energy model as follows:

$$
P_{i}=U_{i} P_{i}^{t s}+\sum_{j=1, j \neq i, S_{j} \notin \widehat{S}}^{n} U_{j} P_{i}^{r s}+\Delta_{i} .
$$

In this example we considered that stream $S_{j} \in \widehat{S}$ either always sends messages to node $i$, or it never sends messages to node $i$, therefore its utilization $U_{j}$ is not considered in the energy model. This is the same as considering $U_{j}=0$. It is worth pointing out that, in several cases a stream $S_{j}$ can only send some messages to node $i$, so the utilization $U_{j}$ has to be recomputed accordingly.

As stated before, to reduce the energy wasted due to the idle listening a node can go in sleep state during idle times and when a message is not addressed to it. Notice that the way the nodes can exploit the idle time, to go in sleep state, depends on the MAC protocol. Moreover, as the idle time could be fragmented into small intervals, and switching between operative modes consumes energy, a node goes in sleep state only if the idle interval is sufficiently long to save energy. Formally, if $t_{i d}$ is the idle fragment length and remembering that $P_{i}^{r s}=P_{i}^{r x}-P_{i}^{s l}$, a node $i$ goes on sleep if and only if:

$$
\begin{gathered}
P_{i}^{r s}\left(t_{i d}-2 t_{s w}\right) \geq 2 P_{i}^{s w} t_{s w} \\
P_{i}^{r s} \geq P_{i}^{s w} \frac{2 t_{s w}}{t_{i d}-2 t_{s w}}
\end{gathered}
$$

The same reasoning applies when a node goes in sleep state because it is receiving a message sent to an other destination. In this case it is sufficient to substitute $t_{i d}$ with the message length in the last inequality.

\section{Problem formulation}

To design our algorithm, the following assumptions are envisaged. At any time, each node is able to monitor its available energy. To save its energy, a node $i$ reduces its transmission power $P_{i}^{t x}$ at the minimum level that guarantees the network connectivity.

On the MAC layer, the communication protocol uses a TDMA scheme to manage the wireless channel. The advantage of this scheme with respect to the carrier sense multi access (CSMA) is that nodes do not waste energy to contend the channel access and there are not packets collisions.

In real-time communication systems, a message stream set $\Gamma$ is said to be feasible if every message in each stream is sent by its deadline.

Several feasibility tests for message stream sets are based on the worst-case achievable utilization (WCAU) of the MAC protocol used to schedule the messages. In general, the WCAU of a scheduling protocol $\mathbf{A}$ represents the largest utilization $U^{*}(\mathbf{A})$ of the network such that, for any real-time stream set whose total network utilization is $U \leq U^{*}(\mathbf{A})$, A can guarantee the timeliness of each single real-time message. 
To simplify the notation, when it is not necessary to specify the protocol, we will write $U^{*}$ instead of $U^{*}(\mathbf{A})$.

Notice that, $U \leq U^{*}$ is generally a sufficient but not necessary condition for the schedulability of $\Gamma$.

In a system with a limited amount of energy, meeting all deadlines is not sufficient to guarantee the application goal. To take energy into account, we extend the definition of feasibility by also considering the system lifetime.

Definition III.1 Given a stream set $\Gamma=\left(S_{1}, S_{2}, \ldots, S_{n}\right)$ and a desired lifetime $L_{\text {net }}^{d}$, a schedule of $\Gamma$ is said to be feasible if the message deadlines are met and the system lifetime $L_{n e t}$ is greater than or equal to $L_{n e t}^{d}$.

From Equation (3) we can observe that, to reduce the energy consumption, besides reducing the transmission powers $P_{i}^{t x}$, we could also increase the sleep time, by reducing the utilization of each node. In accordance with the elastic model presented in Section III-A, we assume that utilizations $U_{i}$ can range in the interval $\left[U_{i}^{\min }, U_{i}^{\max }\right]$.

To summarize, our system can be described as follows:

- An elastic stream set $\Gamma=\left(S_{1}, S_{2}, \ldots, S_{n}\right)$;

- The desired network lifetime $L_{\text {net }}^{d}$;

- The worst case achievable utilization (WCAU) $U^{*}$ of the MAC protocol;

- The vector of the initial available energies $\vec{E}^{0}=$ $\left(E_{1}^{0}, E_{2}^{0}, \ldots, E_{n}^{0}\right)$;

- The transmission power vector $\vec{P}^{t x}=$ $\left(P_{1}^{t x}, P_{2}^{t x}, \ldots, P_{n}^{t x}\right)$;

- The receiving power vector $\vec{P}^{r x}=\left(P_{1}^{r x}, P_{2}^{r x}, \ldots, P_{n}^{r x}\right)$;

- The sleep power vector $\vec{P} s l=\left(P_{1}^{s l}, P_{2}^{s l}, \ldots, P_{n}^{s l}\right)$;

- The switching power vector $\vec{P}^{s w}=$ $\left(P_{1}^{s w}, P_{2}^{s w}, \ldots, P_{n}^{s w}\right)$.

The objective is to maximize the total channel utilization $U$, maintaining $L_{n e t} \geq L_{n e t}^{d}$. In other words, the objective is to solve the following optimization problem:

$$
\begin{aligned}
& \max : \quad U=\sum_{i=1}^{n} U_{i} \\
& \text { s.t. : } \quad P_{i} \leq \frac{E_{i}^{0}}{L_{n e t}^{d}} \quad \forall i=1, \ldots, n \\
& U_{i}^{\min } \leq U_{i} \leq U_{i}^{\max } \quad \forall i=1, \ldots, n \\
& U \leq U^{*}
\end{aligned}
$$

\section{THE ALGORITHM}

The algorithm for solving the problem is based on an extended concept of network overload that also considers the network lifetime. More specifically, the following definition is adopted in this paper.

Definition IV.1 Given a stream set $\Gamma=\left(S_{1}, S_{2}, \ldots, S_{n}\right)$ with utilization $U$ and lifetime $L_{n e t}$, the communication system is considered overloaded if $U>U^{*}$ or $L_{n e t}<L_{n e t}^{d}$.

\section{A. The Elastic MAC algorithm}

The El-MAC algorithm starts by setting $U_{i}=U_{i}^{\max }$, for all streams. Then, if the system is overloaded, the problem is to find a new channel utilization $U^{d}$ such that $U^{d} \leq U^{*}$ and $L_{n e t} \geq L_{n e t}^{d}$.

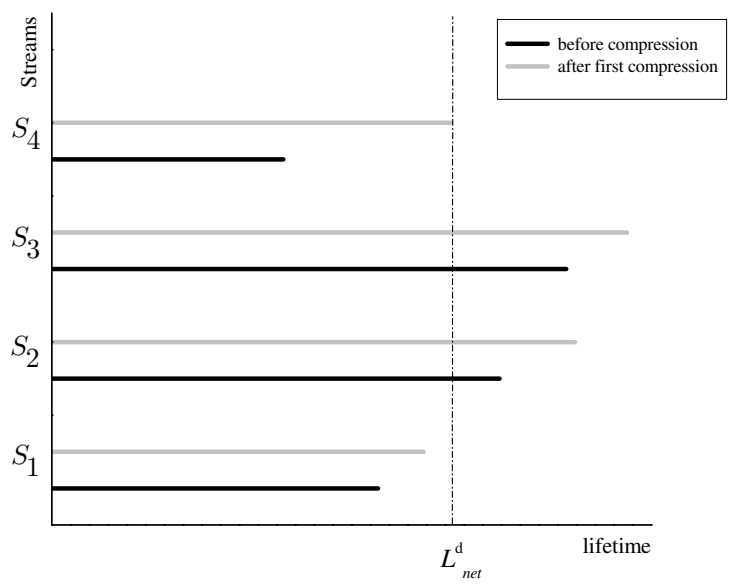

Figure 1. Example on the nodes' lifetime.

If the overload is caused by $U>U^{*}$, the elastic algorithm proposed in [14], can be used to compress the utilizations $U_{i}$ so that $U=U^{*}$. If lifetime is also met under this setting, the algorithm stops returning the utilization vector $\vec{U}=\left(U_{1}, U_{2}, \ldots, U_{n}\right)$. Otherwise, when $L_{n e t}<L_{n e t}^{d}$ if $S_{y}$ is the stream with the shortest lifetime, all stream utilizations are reduced to increase $L_{y}$ up to $L_{n e t}^{d}$. A sample scenario is illustrated in Figure 1, where, before compression, streams $S_{1}$, and $S_{4}$ cannot reach the desired lifetime and $S_{1}$ is the stream with the shortest lifetime less than $L_{n e t}^{d}$, which determines the network lifetime.

To guarantee the lifetime constraint of the network we have to reduce the power consumption of the overloaded streams $\left(S_{1}, S_{4}\right)$. Considering the dependencies expressed in Equation (3), all the stream utilizations are properly reduced to reach the desired network lifetime. In particular, the stream utilizations must be reduced so that, for all $i, U_{i}^{\min } \leq U_{i} \leq U_{i}^{\max }$ and $L_{4}=L_{\text {net }}^{d}$.

Notice that, after such a compression, all stream lifetimes will increase, but the lifetime of some streams could still be less than $L_{n e t}^{d}$. For instance, in the example shown in Figure 1, stream lifetimes after the first compression are indicated by the gray bars, and lifetime of stream $S_{1}$ is still less than $L_{\text {net }}^{d}$. In this condition, the stream with the shortest lifetime is $S_{1}$, thus a new compression must take place to bring $L_{1}=L_{\text {net }}^{d}$. In general, the compression procedure has to be repeated until the network lifetime constraint is satisfied, that is, until $\forall i P_{i} \leq$ $E_{i}^{0} / L_{n e t}^{d}$.

Notice that, at each compression step, stream utilizations are reduced using the elastic algorithm presented in [14], according to which the new utilizations are computed as follows:

$$
U_{i}=U_{i}^{0}-\frac{U^{0}-U^{d}}{\varepsilon} \epsilon_{i}
$$

where $U^{d}$ is the total desired utilization, $U_{i}^{0}$ is the nominal utilization of stream $S_{i}, U^{0}$ is the sum of nominal utilizations, and $\varepsilon=\sum_{i=1}^{n} \epsilon_{i}$. The way to choose the elastic coefficients 
is discussed in Section V.

Note that, in the classical elastic algorithm, the only constraint $U^{d}$ must satisfy is $U^{d} \leq U^{*}$. In this context, however, also the lifetime constraint must be met, hence, $U^{d}$ must also satisfy $L_{n e t} \geq L_{n e t}^{d}$. Considering that the network lifetime is imposed by the stream $\left(S_{y}\right)$ with the shortest lifetime $\left(L_{n e t}=L_{y}\right)$, the nominal power $P_{y}^{0}$ consumed by $S_{y}$ must be decreased to reach the desired lifetime, that is $P_{y}^{d}=E_{y}^{0} / L_{\text {net }}^{d}$.

In the following, the stream power expressed by Equation (3) is combined with Equation (9) to derive the value of $U^{d}$ to be used as a reference in the compression algorithm.

From the energy model (Equation (3)):

$$
\begin{aligned}
\Delta P_{y} & =P_{y}^{0}-P_{y}^{d} \\
& =\left(U_{y}^{0}-U_{y}\right) P_{y}^{t s}+\sum_{j \neq y}^{n}\left(U_{j}^{0}-U_{j}\right) P_{y}^{r s} \\
& +\Delta_{y}^{0}-\Delta_{y} .
\end{aligned}
$$

Using Equation (9), we can substitute $\left(U_{y}^{0}-U_{y}\right)$ and $\left(U_{j}^{0}-\right.$ $U_{j}$ ) in Equation (10) as follows:

$$
\begin{aligned}
\Delta P_{y} & =\left(\frac{U^{0}-U^{d}}{\varepsilon} \epsilon_{y}\right) P_{y}^{t s}+\sum_{j \neq y}^{n}\left(\frac{U^{0}-U^{d}}{\varepsilon} \epsilon_{j}\right) P_{y}^{r s} \\
& +\Delta_{y}^{0}-\Delta_{y} \\
\Delta P_{y} & =\frac{U^{0}-U^{d}}{\varepsilon}\left(\epsilon_{y} P_{y}^{t s}+\sum_{j \neq y}^{n} \epsilon_{j} P_{y}^{r s}\right)+\Delta_{y}^{o l d}-\Delta_{y} \\
\Delta P_{y} & =\frac{U^{0}-U^{d}}{\varepsilon}\left(\epsilon_{y} P_{y}^{t s}+\left(\varepsilon-\epsilon_{y}\right) P_{y}^{r s}\right)+\Delta_{y}^{0}-\Delta_{y} \\
\Delta P_{y} & =\frac{U^{0}-U^{d}}{\varepsilon}\left(\epsilon_{y} P_{y}^{t r}+\varepsilon P_{y}^{r s}\right)+\Delta_{y}^{0}-\Delta_{y}
\end{aligned}
$$

where $P_{y}^{t r}=P_{y}^{t s}-P_{y}^{r s}=P_{y}^{t x}-P_{y}^{r x}$.

From Equation (12) it follows that:

$$
\frac{U^{0}-U^{d}}{\varepsilon}=\frac{\Delta P_{y}-\left(\Delta_{y}^{0}-\Delta_{y}\right)}{\left(\epsilon_{y} P_{y}^{t r}+\varepsilon P_{y}^{r s}\right)} .
$$

Substituting Equation (13) into Equation (9), we have:

$$
U_{i}=U_{i}^{0}-\frac{\Delta P_{y}-\left(\Delta_{y}^{0}-\Delta_{y}\right)}{\epsilon_{y} P_{y}^{t r}+\varepsilon P_{y}^{r s}} \epsilon_{i} .
$$

Notice that, being $\Delta_{y}^{0}-\Delta_{y}$ a small positive quantity, it can safely be neglected still leading to a conservative result. In this case, Equation (14) becomes:

$$
U_{i}=U_{i}^{0}-\frac{\Delta P_{y}}{\epsilon_{y} P_{y}^{t r}+\varepsilon P_{y}^{r s}} \epsilon_{i} .
$$

where $\Delta P_{y}$ is given by:

$$
\Delta P_{y}=P_{y}^{0}-P_{y}^{d}=P_{y}^{0}-\frac{E_{y}^{0}}{L_{n e t}^{d}} .
$$

The overall stream compression algorithm is shown in Figure 2. Summarizing, El-MAC works as follows. The input parameter is the desired lifetime $L_{n e t}^{d}$. The two loops from

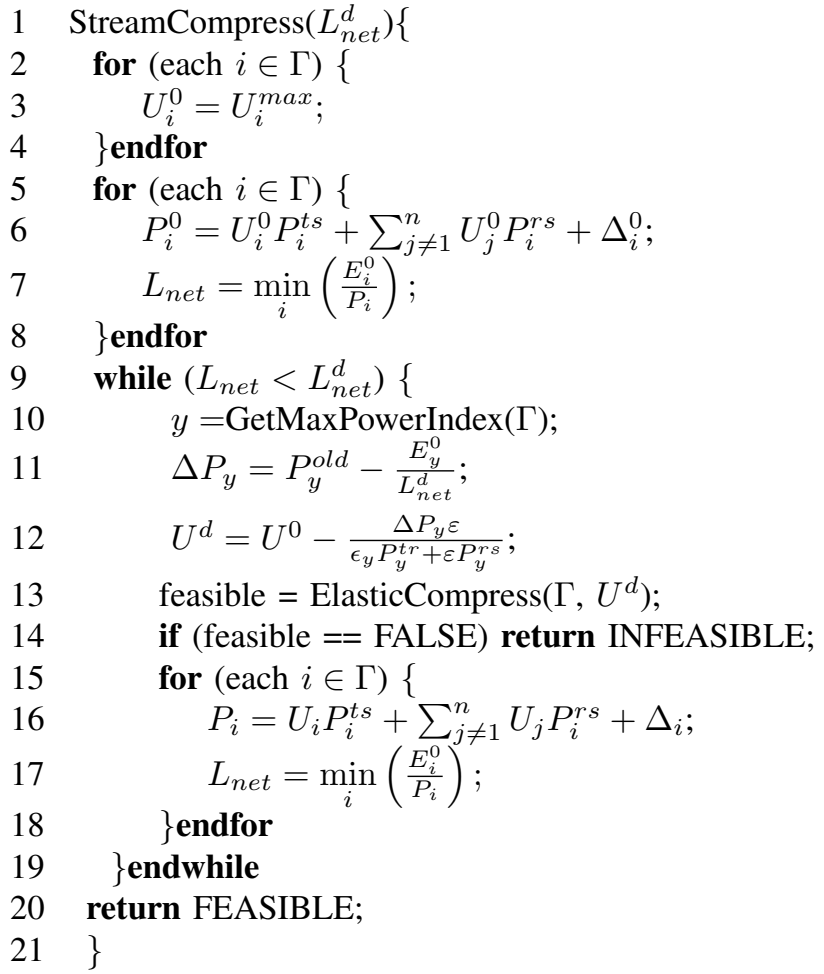

Figure 2. Stream compression algorithm

line 2 to line 8 set the initial stream utilizations and compute the system lifetime $L_{n e t}$. The cycle starting at line 9 and ending at line 19 is the core of El-MAC. First, the index of the stream with lowest lifetime is obtained (line 10), then the desired utilization $U^{d}$ is computed according to Equation (13). $U^{d}$ is passed to the elastic compression procedure defined in [14]. After that, the new system lifetime is calculated and the cycle is repeated until either the lifetime constraint is met $\left(L_{n e t} \geq L_{\text {net }}^{d}\right)$ or the system results to be infeasible $\left(\sum U_{i}^{\text {min }}>U^{d}\right)$.

The complexity of the algorithm in Figure 2 is $O\left(n^{3}\right)$. In fact, in the worst case, all the $n$ streams might be compressed using the elastic procedure, which has an $O\left(n^{2}\right)$ complexity.

\section{B. Applicative example}

To clarify the proposed approach, we describe how to compute the parameters of the energy model (Equation 3), considering the RI-EDF protocol [7], as an example. RI-EDF is MAC layer protocol for cellular structured networks which makes use of a distributed EDF algorithm to schedule node messages. Under RI-EDF, the system time is measured in packets or frames, that is, for each stream $S_{i}$ the message length $C_{i}$, the period $T_{i}$ and the deadline $D_{i}$ are expressed in number of packets. This assumption allows the use of a preemptive algorithm, in that, any preemption is done at packet level, which is considered an atomic unit. Moreover, since each node computes the global channel schedule, any node knows how many packets it can transmit before being preempted by 
a higher priority transmission.

Let us assume a network where nodes are grouped into hexagonal cells, each one working on a different radio channel. The channels are assigned such that co-channel interferences are avoided. Moreover, in each cell any node can listen to any other. Without any loss of generality, we assume each cell being composed by $n-1$ nodes plus a router node which is in charge of inter-cell communication. To each node, including the router, is associated with an elastic stream of periodic messages. For further details on the protocol see the original papers [5], [7].

We can remark that:

- node $i$ is on transmission mode either when delivering its periodic messages, hence $U_{i}=C_{i} / T_{i}$, or when transmitting recovery messages;

- each node knows the schedule of the communication, such that it can compute the bandwidth $U_{i}^{s w}$ wasted switching between operating modes. $U_{i}^{s w}$ can be computed by considering the total number $N_{i}^{s w}$ of mode switches, performed by node $i$ in the hyper-period $H$ (i.e., the least common multiple of the stream periods). Thus, we have: $U_{i}^{s w}=\left(N_{i}^{s w} / H\right) t_{i}^{s w}$.

- the bandwidth that each node $i$ uses to deliver control messages, $U_{i}^{c t r l}$, depends on the number of recovery messages it has to transmit in the hyperperiod $H$. In detail, let $t_{r e c}$ be the time needed to transmit a recovery message, and $N_{i}^{r e c}$ be the number of recovery messages transmitted by node $i$ during $H$ time units, it follows that:

$$
U_{i}^{c t r l}=\frac{N_{i}^{r e c} t_{r e c}}{H}
$$

The value of $N_{i}^{r e c}$ mainly depends on the channel status, e.g. on the packet error rate, which varies during the network operation. Thus, the value of $N_{i}^{r e c}$ can be computed only statistically.

To complete the example, it remains to show how to calculate the value of $U_{i}^{s w}$ for each stream $S_{i}$. Consider for simplicity a network composed by two nodes, associated with a stream $S_{1}\left(C_{1}=3, T_{1}=10\right)$ and $S_{2}\left(C_{2}=2, T_{2}=6\right)$, respectively. The schedule of the channel access by the two nodes is shown in Figure 3. The figure shows both the schedule of the transmission time of each node, and the schedule of the sleep time represented by a dummy node associated with a stream $S_{S l}\left(C_{s l}, T_{S l}\right)$. In particular, remembering that the network nodes go on sleep during the idle time, it turns out that $C_{S l}$ is equal to the idle time during the hyperperiod $H$ and, consequently, $T_{S l}$ is equal to $H$, which in turn is equal to $\operatorname{LCM}\left(T_{1}, T_{2}\right)=30$. The sleep time, $C_{S l}$, can be simply computed as follows:

$$
C_{S l}=H\left(1-\sum_{i=1}^{2} U_{i}\right)=30\left(1-\frac{3}{10}+\frac{2}{6}\right)=11 .
$$

We note that, when node 1 goes on transmission, node 2 goes on receiving mode and viceversa. Furthermore, both nodes go on sleep when the dummy node, associated with stream $S_{S l}$, "access the channel". From these last observations, we note that $N_{1}^{s w}=N_{2}^{s w}=N^{s w}$. Moreover, to calculate $N^{s w}$

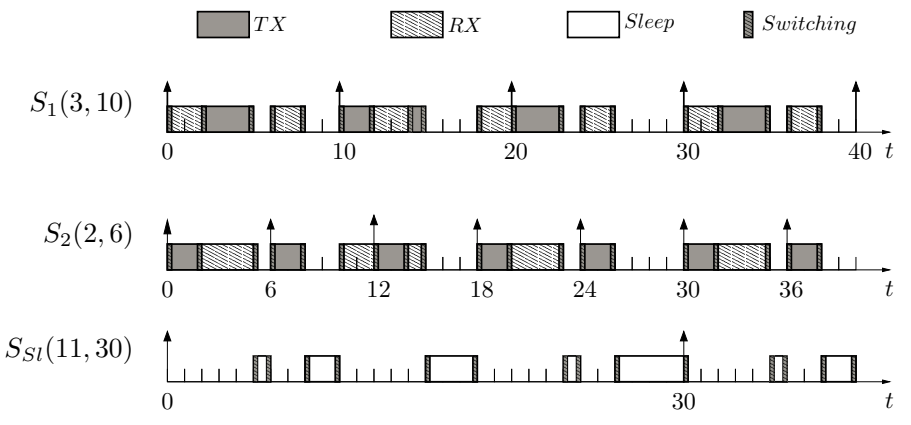

Figure 3. $U_{i}^{s w}$ calculation with RI-EDF.

it is sufficient to count how many times each node, included the sleep node, accesses the channel during the hyperperiod $H=30$. From Figure 3, we see node 1 accesses the channel 4 times, both node 2 and the sleep node 5 times. Thus, it turns out that $N^{s w}=14$ and $U_{1}^{s w}=U_{2}^{s w}=14 / 30=0.467$.

Finally notice that, as stated in Section IV-A we assume that $\Delta_{y}^{0}-\Delta_{y}=0$, hence with El-MAC it is not strictly necessary to calculate $U_{i}^{s w}$ and $U_{i}^{c t r l}$.

\section{AlgorithM PROPERTIES}

In this section we report some observations and results on the El-MAC properties. First, it is worth observing that in general, the proposed algorithm (El-MAC) provides a solution for a more relaxed problem than that defined at the end of Section III (refer to optimization problem (8)). In other words, El-MAC provides an utilizations vector $\vec{U}=\left(U_{1}, U_{2}, \ldots, U_{n}\right)$, such that:

- $U=\sum_{i=1}^{n} U_{i} \leq U^{*}$, that is, the message deadlines are met;

- $\forall i, U_{i}^{\min } \leq U_{i} \leq U_{i}^{\max }$

- $L_{n e t}=L_{n e t}^{\bar{d}}$, that is, the system lifetime is met;

- $U \leq U^{o p t}$;

where $U^{\text {opt }}$ is the solution of the optimization problem (8). The algorithm provides a solution such that the stream set $\boldsymbol{\Gamma}$ is feasible in the sense of Definition III.1, but this solution might not be the optimal one.

Observe that, if the system lifetime is met when for every $i$ $U_{i}=U_{i}^{\text {min }}$, El-MAC certainly produces a solution because, in the worst case, this is the solution provided by the algorithm. Conversely, if the system lifetime is not met with the minimal stream utilizations, no solution exists.

\section{A. Properties of the algorithm parameters}

The following properties are helpful to improve the algorithm performance, especially when it is used in embedded systems where the computation power is limited.

For the sake of clarity, in the remaining part of the paper we consider to have homogenous nodes (nodes with the same radio transceiver), that is, $P_{i}^{r s}=P^{r s}, P_{i}^{t s}=P^{t s}$ for all $i$. We also consider $\Delta_{i} \simeq \Delta$ for all $i$.

The next lemma is used to prove the subsequent theorem.

Lemma V.1 Given a message stream set $\Gamma=$ $\left\{S_{1}, S_{2}, \ldots, S_{n}\right\}$, for any $(i, v)$ with $i \neq v$, if $P^{t s} \geq P^{r s}$ then 
$P_{i} \geq P_{v}$ if and only if $U_{i} \geq U_{v}$. If $P^{t s} \leq P^{r s}$ then $P_{i} \geq P_{v}$ if and only if $U_{i} \leq U_{v}$.

Proof: From the energy model, $P_{i} \geq P_{v}$ means that:

$$
U_{i} P^{t s}+\sum_{j=1, j \neq i}^{n} U_{j} P^{r s}+\Delta \geq U_{v} P^{t s}+\sum_{j=1, j \neq v}^{n} U_{j} P^{r s}+\Delta
$$

then

$$
\begin{gathered}
\left(U_{i}-U_{v}\right) P^{t s}+\left(\sum_{j=1, j \neq i}^{n} U_{j} P^{r s}-\sum_{j=1, j \neq v}^{n} U_{j} P^{r s}\right) \geq 0 \\
\left(U_{i}-U_{v}\right) P^{t s}+\left(U-U_{i}-U+U_{v}\right) P^{r s} \geq 0 \\
\left(U_{i}-U_{v}\right) P^{t s} \geq\left(U_{i}-U_{v}\right) P^{r s}
\end{gathered}
$$

From Inequality (17), if $P^{t s} \geq P^{r s}$, then $P_{i} \geq P_{v}$ if and only if $U_{i} \geq U_{v}$. If $P^{t s} \leq P^{r s}$, then $P_{i} \geq P_{v}$ if and only if $U_{i} \leq U_{v}$.

The following theorem provides a way to select the coefficients $\epsilon_{i}$, in particular the elastic coefficient $\epsilon_{y}$ of stream $S_{y}$, so that the algorithm converges by one iteration, i.e. the cycle (from line 9 to line 19 in Figure 2) is executed just once.

Theorem V.2 Given an elastic stream set $\Gamma=$ $\left\{S_{1}, S_{2}, \ldots, S_{n}\right\}$, let $S_{y}$ be the stream with the shortest lifetime and let $k_{i}=\frac{U_{y}-U_{i}}{U^{0}-U^{d}}$. If $P^{t s} \geq P^{r s}$ then El-MAC provides a solution by just one iteration if and only if for all $i$ :

$$
\epsilon_{y} \leq \epsilon_{i} \frac{1+k_{i}}{1-k_{i}}+\frac{k_{i}}{1-k_{i}} \varepsilon^{i, y}
$$

If $P^{t s} \leq P^{r s}$ El-MAC provides a solution by just one iteration if and only if for all $i$ :

$$
\epsilon_{y} \geq \epsilon_{i} \frac{1+k_{i}}{1-k_{i}}+\frac{k_{i}}{1-k_{i}} \varepsilon^{i, y}
$$

$$
\text { where } \varepsilon^{i, y}=\left(\sum_{j=1 ; j \neq i, y}^{n} \epsilon_{j}\right)=\left(\sum_{j=1}^{n} \epsilon_{j}\right)-\epsilon_{i}-\epsilon_{y} \text {. }
$$

Proof: First we prove the Inequality (18).

(If) Suppose that the algorithm converges by one iteration, that is, after the first iteration $P_{y}^{d}=E_{i}^{0} / L_{n e t}^{d}$ and $\forall i P_{y}^{d} \geq P_{i}$. From Lemma V.1, $\forall i$ if $P^{t s} \geq P^{r s}$ then $P_{y}^{d} \geq P_{i}$ if and only if $U_{y} \geq U_{i}$, that is, $U_{y}-U_{i} \geq 0$. From Equation (9),

$$
\begin{gathered}
U_{y}-U_{i}=U_{y}^{0}-U_{i}^{0}-\frac{U^{0}-U^{d}}{\varepsilon}\left(\epsilon_{y}-\epsilon_{i}\right) \geq 0 \\
U_{y}^{0}-U_{i}^{0} \geq \frac{U^{0}-U^{d}}{\varepsilon}\left(\epsilon_{y}-\epsilon_{i}\right) \\
\frac{U_{y}^{0}-U_{i}^{0}}{U^{0}-U^{d}} \varepsilon \geq\left(\epsilon_{y}-\epsilon_{i}\right)
\end{gathered}
$$

Since $\varepsilon=\sum_{j=1}^{n} \epsilon_{j}=\varepsilon^{i, y}+\epsilon_{i}+\epsilon_{y}$, and $k_{i}=\frac{U_{y}^{0}-U_{i}^{0}}{U^{0}-U^{d}}$, then if the algorithm ends by one iteration:

$$
\begin{array}{r}
\left(\epsilon_{y}-\epsilon_{i}\right) \leq k_{i}\left(\left(\sum_{j \neq i, y} \epsilon_{j}\right)+\epsilon_{i}+\epsilon_{y}\right) \\
\epsilon_{y}\left(1-k_{i}\right) \leq \epsilon_{i}\left(1+k_{i}\right)+k_{i} \varepsilon^{i, y} \\
\epsilon_{y} \leq \epsilon_{i} \frac{1+k_{i}}{1-k_{i}}+\frac{k_{i}}{1-k_{i}} \varepsilon^{i, y}
\end{array}
$$

(Only if) Suppose that the algorithm ends by one iteration and for some $i, \epsilon_{y}>\epsilon_{i} \frac{1+k_{i}}{1-k_{i}}+\frac{k_{i}}{1-k_{i}} \varepsilon^{i, y}$, then following the inverse process we find out that in this case $U_{y}<U_{i}$, that is $P_{y}^{d}=E_{i}^{0} / L_{\text {net }}^{d}<P_{i}$, hence the algorithm has not terminated, this is a contradiction, therefore the thesis follows.

Now, suppose that the algorithm converges by one iteration, hence $P_{y}^{d}=E_{i}^{0} / L_{\text {net }}^{d}$ and $\forall i P_{y}^{d} \geq P_{i}$. To prove the Inequality (19) it is sufficient to observe that, from Lemma V.1, if $P^{t s} \leq P^{r s}$ then $P_{y}^{d} \geq P_{i}$ if and only if $U_{y} \leq U_{i}$, that is, $U_{y}-U_{i} \leq 0$. The rest of the prove is similar to that for the Inequality (18), where it is sufficient to exchange the signs $(\leq)$ and $(\geq)$.

It is worth noticing that, from Inequality (18), if for some $i k_{i}>1, P^{t s} \geq P^{r s}$ then $\epsilon_{y}<0$. This means that, we have no solutions because $\epsilon_{y}$ must be non negative. Further, from Inequality (19), if $k_{i}=1$ and $P^{t s} \leq P^{r s}$ we have no solutions because $\epsilon_{y} \geq \infty$.

The following corollary guarantees that an available solution for $\epsilon_{y}$ always exists, in other words, it guarantees that the bad conditions described above are impossible.

Corollary V.3 Given an elastic stream set $\Gamma=$ $\left\{S_{1}, S_{2}, \ldots, S_{n}\right\}$, let $S_{y}$ be the stream with the shortest lifetime, defined $k_{i}=\frac{U_{y}^{0}-U_{i}^{0}}{U^{0}-U^{d}}$, if $P^{t s} \geq P^{r s}$ then for any $i$ $0 \leq k_{i} \leq 1$. If $P^{t s} \leq P^{r s}$ then for any $i k_{i} \leq 0$.

Proof: We start defining a function $f_{\epsilon}\left(k_{i}\right)=\epsilon_{i} \frac{1+k_{i}}{1-k_{i}}+$ $\frac{k_{i}}{1-k_{i}} \varepsilon^{i, y}$, where $i \neq y$, and $\epsilon_{i}, \varepsilon^{i, y}$ are given parameters.

In the first case, where $P^{t s} \geq P^{r s}$, from Lemma V.1, at each step the algorithm chooses $y$ so that for all $i, U_{y}^{0} \geq U_{i}^{0}$, then $k_{i} \geq 0$. we can observe that:

- From Theorem V.2, if $k_{i}<1$ then to converge by just one iteration we need that $\epsilon_{y} \leq f_{\epsilon}\left(k_{i}\right)<\infty$. For $k_{i}=1$, $f_{\epsilon}\left(k_{i}\right)=\infty$. Thus, we can choose $\epsilon_{y} \leq f_{\epsilon}\left(k_{i}\right) \leq \infty$ for $0 \leq k_{i} \leq 1$.

- If $k_{i}>1$, from Inequality (18), to converge by just one iteration we need that $\epsilon_{y} \leq f_{\epsilon}\left(k_{i}\right)<0$. Note that, being $\epsilon_{y}$ an elastic coefficient, $\epsilon_{y}<0$ has no meaning. However, it is not difficult to see that if for all $i$, $0 \leq \epsilon_{y} \leq \epsilon_{i}$ then $U_{y} \geq U_{i}$, that is: the algorithm finishes by just one iteration. Therefore, being the Inequality (18) a necessary and sufficient condition, it follows that either $0 \leq k_{i} \leq 1$ or Inequality (18) is incorrect. Since we proved the correctness this latter inequality, the first part of the thesis follows.

In the second case we have $P^{t s} \leq P^{r s}$. From Lemma V.1 at each step the algorithm chooses $y$ so that for all $i, U_{y}^{0} \leq U_{i}^{0}$, this means that $k_{i} \leq 0$. 


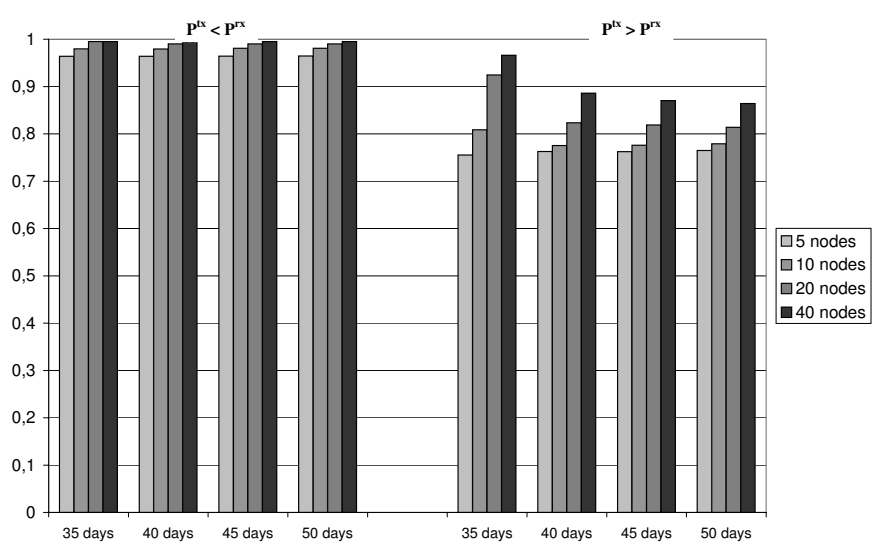

Figure 4. El-MAC performance.

From Theorem V.2, in order to get the coefficients $\epsilon_{i}$, it is necessary to solve a linear inequalities system. However, as the function $f_{\epsilon}\left(k_{i}\right)=\epsilon_{i} \frac{1+k_{i}}{1-k_{i}}+\frac{k_{i}}{1-k_{i}} \varepsilon^{i, y}$ is increasing monotonic for $0 \leq k_{i}<1$ and decreasing monotonic for $k_{i}<0$, once we chose each $\epsilon_{i}$ except $\epsilon_{y}$, defined $k_{\text {min }}=\min _{i}\left(k_{i}\right)$ and $k_{\max }=\max _{i}\left(k_{i}\right)$, it is sufficient to choose $\epsilon_{y}$ as indicated by the Inequalities $(18,19)$, solving just one inequality, considering only $k_{\min }$ and $k_{\max }$ respectively. In this way, the relations of the Theorem V.2 can be satisfied without solving a linear inequalities system.

\section{Simulation Results}

To test the algorithm we performed several simulation experiments varying the main input parameters. We tested El-MAC varying the desired system lifetime (measured on days), the number of nodes involved in the network, and the transceivers used. In particular, we have considered two communication devices, the $C C 2400$ [1] and DR3300 [2], mainly for the fact that the former has $P^{t x}<P^{r x}$ and the latter has $P^{t x}>P^{r x}$. To calculate $P^{t x}$ and $P^{r x}$ we have obtained the operating voltage and the currents sunk in the various working modes from their data sheets.

The following upper bound is used to evaluate the algorithm. From the energy model, expressing $U_{i}$ as function of $P_{i}$ and $U_{j}$, for all $i=1, \ldots, n$ and $j=1, \ldots, n$ with $j \neq i$, we can derive a necessary condition on $U$ :

$$
U_{i}=\frac{P_{i}-\sum_{j \neq i}^{n} U_{j} P^{r s}-\Delta}{P^{t s}} .
$$

Summing up for all $i$ both side of the equation, and remembering that $U=\sum_{i} U_{i}$, we have:

$$
U=\frac{\sum_{i=1}^{n} P_{i}-(n-1) U P^{r s}-n \Delta}{P^{t s}} .
$$

Then, defining $P_{t o t}=\sum_{i=1}^{n} P_{i}$, and gathering the terms:

$$
U=\frac{P_{t o t}-n \Delta}{P^{t s}+(n-1) P^{r s}} .
$$

Let $P_{\text {tot }}^{\max }=\sum_{i=1}^{n} \frac{E_{i}^{0}}{L_{n e t}^{d}}$ be the maximum power that the system can waste while guaranteeing the desired lifetime, we define the following channel utilization upper bound:

$$
U^{u b}=\frac{P_{t o t}^{\max }-n \Delta}{P^{t s}+(n-1) P^{r s}} .
$$

To meet $L_{n e t}^{d}$, a necessary but not sufficient condition is that $P_{\text {tot }} \leq P_{\text {tot }}^{\max }$. From Equations (20) (21), it can be seen that condition $P_{t o t} \leq P_{t o t}^{\max }$ is equivalent to condition $U \leq U^{u b}$. In this way, we found out an upper bound for $U^{d}$. In other words, let $U^{o p t}$ be the solution of Problem (8), it turns out that $U^{d} \leq U^{o p t} \leq U^{u b}$.

The algorithm has been evaluated calculating the performance ratio $p_{\text {ratio }}$ between the total utilization $U^{d}$, provided by El-MAC, and the theoretical upper limit $U^{u b}$ :

$$
p_{\text {ratio }}=\frac{U^{d}}{U^{u b}} .
$$

The simulation was performed generating, for each stream $S_{i}$, the utilizations $U_{i}^{\text {max }}$ and $U_{i}^{\text {min }}$ using the method proposed by Bini et al. [17]. The maximum total utilization, i.e. the sum of the $U_{i}^{\max }$, was equal to $U^{*}$ for each simulation run. In our simulations, we considered a MAC protocol having $U^{*}=1$; an example of such a protocol is RI-EDF (see Section II).

Usually, in the elastic model the coefficients $\epsilon_{i}$ represent the relative importance of each stream. For instance, some traffic streams can be compressed a little because are important for the system operation, some other could be less important hence they can be compressed more. In general, the importance of a stream depends on the application hence, since the evaluation of El-MAC is done without considering any particular application, the elastic coefficients $\epsilon_{i}$ have been generated as random numbers with uniform distribution in $[0,1]$.

As mentioned above, each experiment set was built selecting three parameters: the number of nodes $n$, the desired system lifetime $L_{n e t}^{d}$, and the transceiver. For each experiment set, the algorithm was simulated 1000 times, generating $U_{i}^{\max }$ and $U_{i}^{\text {min }}$ for each run, providing the average value of $p_{\text {ratio }}$. The algorithm has been implemented using MATLAB [18].

Figure 4 shows on the left side the results obtained with the transceiver $C C 2400$, having $P^{t x}<P^{r x}$. The same figure shows on the right side the results with the transceiver $D R 3300$, having $P^{t x}>P^{r x}$. The figure shows the performance ratio under different desired system lifetimes (days) and number of nodes $n$. Experiments are grouped by $L_{n e t}^{d}$ measured in days, and each element in a group represents the performance ratio for a specific number of nodes in the network.

As we can see, for $P^{t x}<P^{r x}$, fixing $n$ and increasing $L_{n e t}^{d}, p_{\text {ratio }}$ presents a very small variation. Instead, by fixing $L_{n e t}^{d}$ and varying $n, p_{\text {ratio }}$ grows up to 1 . In other words, from the definition of the performance ratio, by increasing the number of nodes the algorithm produces a solution closer to the channel upper bound $U^{u b}$; that is, the solution tends to be optimal. Increasing the desired lifetime, in practice the algorithm performance does not change. It is worth noticing that, when $P^{t x}<P^{r x}$, the performance ratio is very close to 1 in all simulated scenarios. 
For the case $P^{t x}>P^{r x}$, for $n$ equal to 20 and 40 , varying $L_{\text {net }}^{d}$ the performance ratio decreases. This last remark is not valid for $n$ equal to 5 and 10 , because in this case $p_{\text {ratio }}$ remains more or less the same when $L_{n e t}^{d}$ changes.

Fixing $L_{\text {net }}^{d}$, the performance ratio $p_{\text {ratio }}$ increases with $n$. In the second scenario $\left(P^{t x}>P^{r x}\right)$, the solutions provided by the algorithm are characterized by a lower performance ratio with respect to the first setting in which $P^{t x}<P^{r x}$.

\section{CONCLUSION AND FUTURE WORK}

In this paper we analyzed the problem of saving energy at the communication level for a distributed embedded system, formed by a network of wireless nodes. First, we described the main sources of energy waste regarding a wireless network composed by distributed devices. We assumed that each node is associated with a message stream, described through an elastic stream model in which each stream utilization (bandwidth) can vary in a given interval. Given the available energy on each node, we derived an energy model that allows estimating the network lifetime. This model takes into account the energy wasted to deliver messages by the radio transceiver. This energy is managed at the MAC level.

After having defined the system lifetime, we introduced an algorithm (El-MAC) to select the bandwidth of each stream within a specified range, so that both real-time constraints and the network lifetime can be guaranteed. We also showed that, if the elastic coefficients of the streams are appropriately chosen, the algorithm ends by just one iteration.

We tested El-MAC by simulation, using the parameters provided by the datasheets of two different radio transceivers. The first one with $P^{t x}>P^{r x}$ and the second one with $P^{t x}<P^{r x}$. We used as performance metric a theoretic upper bound on the channel utilization $U^{u b}$, which is an upper bound for a solution of the optimization problem (8), introduced in Section III-C.

The proposed energy model and El-MAC are directly applicable to TDMA scheduling approaches, as for instance the IEDF protocol. The major differences among the various scheduling protocols can reside in the computation of $U^{s w}$ and $U^{c t r l}$, which strictly depend on the MAC protocol adopted.

As a future work, we plan to devise an algorithm able to produce a solution to the optimization problem described in Section III-C through the Equation (8).

\section{REFERENCES}

[1] Chipcon CC2420, http://www.ti.com

[2] Rfm DR3300, http://www.rfm.com.

[3] W. Ye, J. Heidemann, and D. Estrin, "Medium access control with coordinated adaptive sleeping for wireless sensor networks," IEEE/ACM Transaction on networking, vol. 12, no. 3, pp. 493-506, June 2004.

[4] M. Adamou, I. Lee, and I. Shin, "An energy efficient real-time medium access control protocol for wirelss ad-hoc networks," in Proc. of the 22th IEEE Real-Time Systems Symposium (RTSS 2001).

[5] M. Caccamo, L. Y. Zhang, L. Sha, and G. Buttazzo, "An implicit prioritized access protocol for wireless sensor networks," in Proc. of the IEEE Real-Time Systems Symposium (RTSS 2002), Dec. 2002.

[6] C. L. Liu and J. W. Layland, "Scheduling algorithms for multiprogramming in a hard-real-time environment," Journal of the ACM (JACM), vol. 20, no. 1, pp. 46-61, Feb. 1973.
[7] T. L. Crenshaw, A. Tirumala, S. Hoke, and M. Caccamo, "A robust implicit access protocol for real-time wireless collaboration," in Proc. of the IEEE Euromicro Conference on Real-Time Systems (ECRTS 2005), Jul. 2005.

[8] T. He, J. A. Stankovic, C. Lu, and T. F. Abdelzaher, "SPEED: A stateless protocol for real-time communication in sensor networks," in Proceedings of the International Conference on Distributed Computing Systems (ICDCS 2003).

[9] J. A. Stankovic, T. He, T. F. Abdelzaher, M. Marley, G. Taoand, S.Son, and C. Lu, "Feedback control scheduling in distributed systems," in Proc. of the 22th IEEE Realt-Time Systems Symposium (RTSSO1), London, UK, Dec. 2001.

[10] A. Koubaa, M. Alves, and E. Tovar, "Energy/Delay Trade-off of the GTS Allocation Mechanism in IEEE 802.15.4 for Wireless Sensor Network," International Journal of Communication Systems, vol. 20, no. 7, pp. 791-808, July 2007.

[11] A. Rowe, R. Mangharam, and R. Rajkumar, "RT-Link: A TimeSynchronized Link Protocol for Energy-Constrained Multi-hop Wireless Networks," in Third IEEE Int. Conf. on Sensors, Mesh and Ad Hoc Communications and Networks (IEEE SECONO6).

[12] D. D. Demarch and L. B. Becker, "An integrated scheduling and retransmission proposal for firm real-time traffic in ieee 802.11e." in Proc. of the IEEE Euromicro Conference on Real-Time Systems (ECRTS 2007), Jul. 2007.

[13] M. M. Sobral and L. B. Becker, "A wireless hybrid contention/tdma based mac for real-time mobile application." in Proc. of the ACM Symposium on Applied Computing (SAC 2008), March. 2008.

[14] G. Buttazzo, G. Lipari, M. Caccamo, and L. Abeni, "Elastic scheduling for flexible workload management," IEEE Transactions on Computers, vol. 51, no. 3, pp. 289-302, March 2002.

[15] Y. Chen and Q. Zhao, "On the lifetime of wireless sensor networks," IEEE Communication Letters, vol. 9, no. 11, pp. 976-978, November 2005.

[16] D. M. Blough and P. Santi, "Investigating upper bounds on network lifetime extension for cell-based energy conservation techniques in stationary ad hoc networks," in Proceedings of MOBICOM 2002.

[17] E. Bini and G. Buttazzo, "Biasing effects in schedulability measures," in Proc. of the 16th Euromicro Conference on Real-Time Systems (ECRTS 2004), June 2004.

[18] MATLAB, http://www.mathworks.com. 